REVIEW

\title{
Advances in paediatric tumour imaging
}

\author{
L Moon, K McHugh
}

Arch Dis Child 2005;90:608-611. doi: 10.1136/adc.2004.051193

There have been a number of major advances in imaging technology in recent years. Here we discuss in detail multislice CT and PET, since these are the two modalities generating the most interest in cancer imaging, but which also have the potential for significantly increasing the radiation burden in the paediatric population. In addition, we focus on the implications of the new Response Evaluation Criteria In Solid Tumours (RECIST) guidelines, which describe a significant new method of tumour size measurement.

See end of article for authors' affiliations

.....................

Correspondence to: Dr L Moon, Radiology Department, Barts and the London Hospitals NHS Trust, London, UK; Ifrosł@ doctors.org.uk

Accepted 25 January 2005
$\mathrm{T}$ his paper will focus on two technological advances in radiological imaging, namely multislice computed tomography (CT) and positron emission tomography (PET), and also on a significant new method of tumour size measurement.

New Response Evaluation Criteria In Solid Tumours (RECIST) guidelines have been devised to assess the response to treatment in solid tumours, with the stated aim of simplifying and standardising tumour measurements. Some problems with regard to paediatric oncology practice remain, and these will be outlined below.

There have been a number of major advances in imaging technology in recent years, but multislice CT and PET are the two new modalities generating most interest in cancer imaging. Unfortunately both these modalities carry a significant radiation burden. It must be remembered that children are significantly more sensitive to the cancer inducing effects of radiation than adults, ${ }^{1}$ and therefore it is particularly important to use these techniques sparingly in young patients, including those with malignant lesions. Consequently, ultrasound and magnetic resonance imaging, neither of which involves ionising radiation, should ideally be the major imaging modalities used in paediatric oncology with the newer techniques providing additional information where necessary. Such radiological developments come at a time when improvements in survival from childhood cancer have increasingly led to the development of risk adapted treatment regimens, with a view to reducing long term morbidity following treatment. These factors have made accurate assessment of tumour load at presentation and follow up paramount. Consequently, an understanding of how these new techniques may aid diagnosis, staging, and affect management of paediatric tumours is important.

\section{RESPONSE EVALUATION CRITERIA IN SOLID TUMOURS}

In 1979 the World Health Organisation (WHO) popularised four specific criteria for the classification of response in solid tumours. ${ }^{2}$ These categories, called complete response (CR), partial response (PR), stable disease (SD), and progressive disease (PD) came to be used widely in both adult and paediatric oncology practice. Problems with these definitions gradually became apparent, and a situation arose whereby the response criteria were no longer comparable between research organisations - the very circumstance that the original WHO publication had set out to avoid. ${ }^{3}$ Consequently WHO, the European Organisation for Research and Treatment of Cancer, the National Cancer Institute of the United States, and the National Cancer Institute of Canada Clinical Trials Group set up a task force in the early 1990s with the objective of again attempting to unify response assessments. Eventually a revised version of the WHO criteria was produced and circulated. ${ }^{3}$

The RECIST document was created as a result of the discussions above. The RECIST criteria continue to rely on size change of lesions to make response assessment. The four response categories (CR, PR, SD, and PD) have been retained to enable comparison of previous and future treatments. Under RECIST, the methods by which lesions are assessed have changed, however. Rather than a bidimensional measurement of lesion area under the old WHO system, a simple unidimensional measurement of maximum diameter is now used, ${ }^{23}$ although no studies to date validating single measurement approach have been done as yet in childhood malignancies. Precision in measurement has been deemed less important than standardised methodology.

The definitions of the response assessment categories have also altered slightly. CR and SD remain the same under RECIST as with the WHO system. To achieve PR the RECIST document requires that there should be at least a 30\% decrease in the longest diameter for a single lesion or in metastatic cases the sum of the longest diameters (LD). ${ }^{2}$ This is roughly equivalent to the previous WHO 50\% reduction in the product of the diameters. The only truly significant change in definition has occurred with

Abbreviations: $C R$, complete response; $\mathrm{CT}$, computed tomography; FDG, fluoro-2-deoxy-D-glucose; MIBG, metaiodobenzylguanidine; $M R I$, magnetic resonance imaging; PD, progressive disease; PET, positron emission tomography; PR, partial response; RECIST, Response Evaluation Criteria In Solid Tumours; SD, stable disease; WHO, World Health Organisation 
the PD category, whereby greater volume increases are now necessary to define disease progression. To define PD, the RECIST criteria require a $20 \%$ increase in diameter for a single lesion or in the sum of the LDs of multiple masses (PD is also defined by the appearance of new malignant lesions but this was also the case with the old WHO system). The need for greater tumour burden to define PD arose because of the relative ease by which a patient could have been mistakenly considered to have disease progression by the WHO criteria.

From the paediatric perspective, the new RECIST guidance places too much emphasis on repeated CT scanning. This excess reliance on CT is unfortunate as the high inherent radiation burden from $\mathrm{CT}$ precludes its repeated use in children, and here the guidance will need to be modified. ${ }^{4}$ The use of ultrasound is discouraged by RECIST because it is an operator dependent technique. Ultrasound is however permitted as an alternative to clinical measurements for thyroid nodules, subcutaneous lesions, and "superficial" palpable lymph nodes. Ultrasound is undoubtedly the modality best suited to repeated scanning of abdominal, and many other, masses in small children-avoiding the hazards of repeated irradiation, sedation, and general anaesthesia.

The minimum lesion size to be measured following a given CT examination is recommended by RECIST to be double the slice thickness obtained during that CT scan. This means that with an old conventional CT scanner, $20 \mathrm{~mm}$ minimum diameter is the lesion size considered to be measurable; however CT scanning has moved on significantly since the RECIST guidance was formulated in the early 1990s, and modification that allows measurements of smaller tumours should now be possible with newer multislice CT scanners. RECIST also stipulates that the LD of each so-called target lesion should be selected from CT images in the axial plane only, but this arbitrary restriction is clearly now outdated. ${ }^{5}$ When magnetic resonance imaging (MRI) is used, wherever possible the same MR scanner should be used when following up patients, with the same magnet strength and sequences. The reality, however, is that some children get initially worked up in their local hospital and are then transferred to a paediatric oncology centre.

Nuclear medicine's contribution to staging of malignancies is ignored by RECIST, as the criteria are primarily concerned with tumour size change. In a new European high risk neuroblastoma study however, a 50\% response in the metaiodobenzylguanidine (MIBG) evaluation is one of the main criteria for randomisation prior to myeloablative therapy following induction chemotherapy.

When the RECIST document was being formulated, there was a regrettable lack of consultation with the paediatric radiological and oncological community. The guidelines with regard to CT will somehow need to be modified for paediatric oncology to take into consideration repeated, and too frequent, irradiation, and also a potentially excessive need for sedation and anaesthesia. Most significantly, although discouraged by the RECIST guidance, ultrasound is the main imaging modality in paediatrics and a greater role for ultrasound in paediatric cancers will somehow need to be agreed. Until new functional imaging techniques with MRI, and particularly PET scanning, become more reliable and widespread it seems likely that these RECIST criteria are going to be relied on for some time as the major indices of response assessment in all age groups.

\section{MULTISLICE CT}

Since the launch of the first multislice (or multidetector array) CT scanners in 1998, their acceptance into clinical

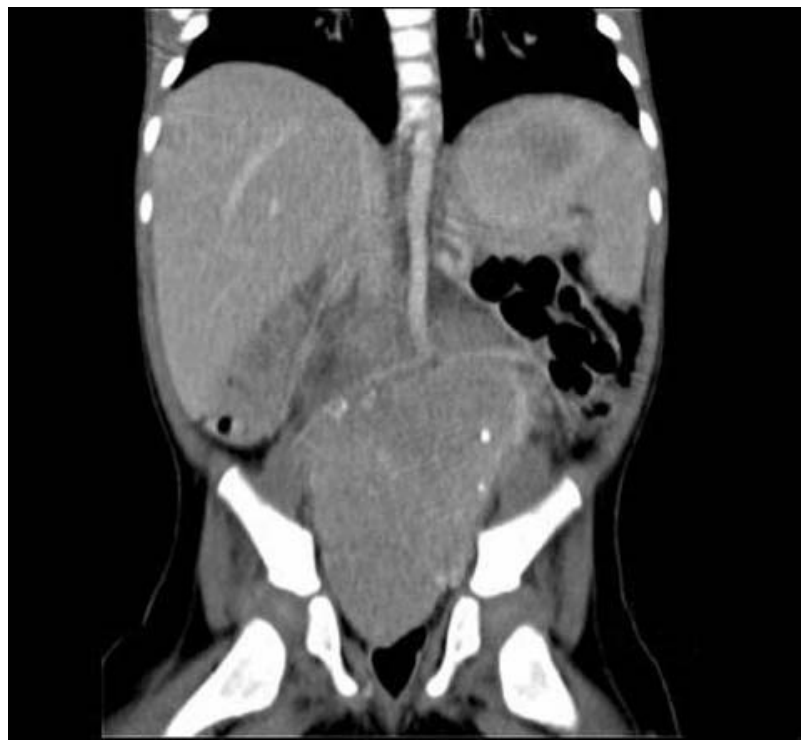

Figure 1 Coronal reconstruction of a CT scan, showing a neuroblastoma widely splaying the aortic bifurcation.

practice has been rapid and widespread, and their use confers several distinct advantages.

More detectors means that fewer $x$ ray tube rotations are required to cover the same volume of the patient and therefore data can be acquired more quickly, or alternatively finer sections can be obtained without increasing the scanning time. A concurrent technical development utilised on multislice scanners is the increased rotation speed of the $x$ ray tube and detector assembly, which in conjunction with much larger volumes covered in a single rotation results in faster overall scanning times. ${ }^{6}$ These features are of relevance in paediatrics because a short scan time may allow more examinations to be performed without sedation or general anaesthesia. ${ }^{7}$ This may confer an advantage over MRI in some children, although MRI remains the technique of choice for some areas such as brain, spinal cord, and bone marrow imaging. Furthermore, most paediatric CT imaging is performed during normal tidal breathing, and the reduced scanning time significantly decreases the respiratory artefact affecting the lungs in particular.

On older single slice scanners the minimum slice width was generally $1 \mathrm{~mm}$. On multislice systems however, because the $x$ ray beam is divided between a number of detectors, slice widths as low as $0.5 \mathrm{~mm}$ can be obtained. The ability to produce such thin slices has introduced the concept of isotropic imaging, such that resolution in all three planes is similar and whereby slice thickness approaches in-slice pixel size. This allows reconstruction in multiple planes without any significant distortion, avoiding the "stepping" effect seen in reconstructions using greater slice thicknesses. In oncology this can be extremely useful in defining more precisely the relations of a mass, since conventional CT has always been weak in the craniocaudal direction, for example showing whether a mass invades the liver, or if a mass that appears suprarenal actually arises from the kidney or adrenal gland. High speed imaging also allows high quality CT angiography, and the relation of a tumour to adjacent vessels in multiple planes can be of great help when planning surgery ${ }^{8}$ (fig 1). Particularly for large infiltrative tumours, comparison of tumour load before and after treatment remains easier with CT than ultrasound due to inter-operator variability inherent with ultrasound, and multislice CT can allow assessment in all planes. 
Thinner slice widths do however result in a greater overall radiation dose to the patient, although because of the arrangement of detectors in a multi slice CT scanner, this is relatively smaller than would be the case with conventional single slice scanners. Doses may, however, be generally higher since collimation of $1 \mathrm{~mm}$ or less was very rarely used in single slice scanners, but frequently used with a multislice system. The ability to perform multiphase contrast studies may also lead to increased dose, although examinations with multiple acquisitions are not commonplace in paediatrics. It has been argued that more accurate scanning, with less patient movement and well timed contrast enhancement will reduce the need for repeat scanning, and hence overall population dose, but this remains to be seen. It is still essential to change standard scanning parameters set for the adult population when scanning younger patients in order to account for the smaller size of children. Unless this adjustment for weight and age is done, children receive unnecessarily high irradiation with no benefit. There are methods of dose reduction in many new multislice CT units such as online tube current modulation, whereby the $x$ ray tube current, and therefore the dose, is reduced dynamically where the patient diameter is smaller, without a loss of image quality. ${ }^{9}$ Dose limitation is of prime importance in paediatric imaging, ${ }^{10}$ and thin slice widths should only be used if they will provide valuable additional information.

Multislice systems produce an extremely large number of images, and new ways of analysing the images, including investment in a workstation to view and manipulate the images is essential. Further refinements such as virtual CT endoscopy and bronchoscopy to assess intraluminal lesions are also easily achieved.

\section{POSITRON EMISSION TOMOGRAPHY (PET)}

Radionuclide functional imaging has become a central part of oncological practice; for example, the use of MIBG is critical

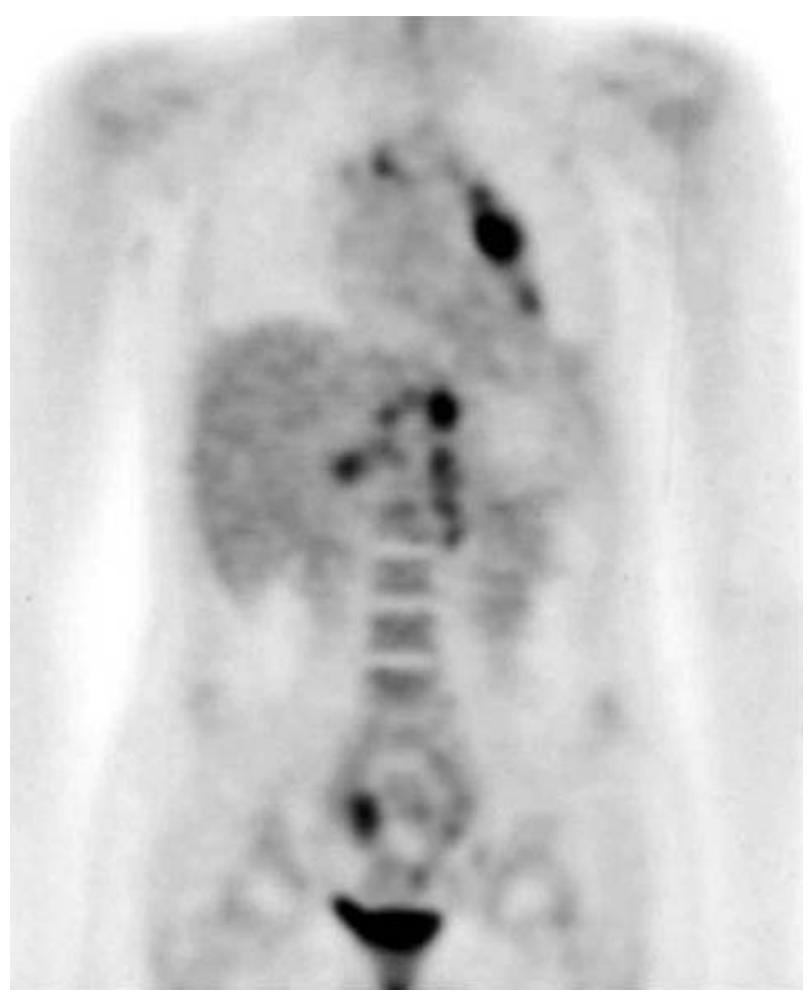

Figure 2 PET scan in the coronal plane, in a patient with known mediastinal lymphoma, and previously unsuspected extensive abdominal and pelvic disease. to the staging in neuroblastoma. PET is one of the most advanced of these modalities, and its use in paediatrics is growing. PET can provide metabolic information to supplement morphological or anatomical imaging, and different tracers will give differing information depending on how tissues handle them.

The history of PET in oncology began with the initial key discovery that cancer cells derive considerably more of their energy from anaerobic glycolysis than normal cells. ${ }^{11}$ It was subsequently shown that a variety of animal tumours concentrate 2 - $\left({ }^{18} \mathrm{~F}\right)$ fluoro-2-deoxy-D-glucose $\left({ }^{18} \mathrm{~F}\right.$-FDG $),{ }^{12} \mathrm{a}$ glucose analogue, and this has become the most commonly used tracer. It has a half life of 112 minutes and must be produced in a cyclotron. For practical purposes the PET camera should not be too remotely located from a cyclotron unit, of which there are currently a limited number in the UK. In order to use other types of tracer such as carbon-11, nitrogen-13, or oxygen-15 (short half lives of 20, 10, and 2 minutes respectively) it is necessary to have a cyclotron and radionuclide laboratory on site, and as a result these agents are probably best regarded as research tools at present (fig 2).

Spatial resolution is currently in the order of $4-5 \mathrm{~mm}$. This is lower than many other imaging techniques, although PET has other advantages. In paediatric lymphoma PET already plays an important role in staging, evaluating tumour response, planning radiotherapy, and monitoring for relapse in some centres (fig 2). Over the last decade PET has gradually replaced gallium-67 as the preferred functional imaging modality for lymphoma staging because the day 1 scan is of higher resolution, is of lower dose, and has less confounding intestinal activity. ${ }^{13}$ Early reports of prospective studies evaluating PET-FDG in paediatric lymphoma have suggested that it may indeed be a useful tool for staging and follow up, although not without limitations. ${ }^{14}$

PET can be useful in distinguishing benign from malignant tumours, may help selection of a biopsy site, and in distinguishing scar from residual neoplasm in children who have completed therapy. ${ }^{15}{ }^{16}$ Regression of metabolic tumour activity detected by PET may serve as a surrogate for tumour response. Most experience in the use of PET has been in imaging lymphoma and brain tumours, although it has shown potential in osteosarcoma, rhabdomyosarcoma, Ewing's sarcoma, ${ }^{16}$ and neuroblastoma, including those 10 $20 \%$ that fail to concentrate MIBG since ${ }^{18}$ F-FDG uptake does not rely on type 1 catecholamine uptake. PET images are not affected by metallic artefacts from limb salvage prostheses, unlike in MRI and CT, and this could be useful for posttreatment follow-up of osteosarcomas, for example. Unfortunately some normal tissues and non-malignant disease processes also show ${ }^{18} \mathrm{~F}-\mathrm{FDG}$ avidity, providing pitfalls for the unwary. "Brown" fat of cervical musculature and muscles can all show marked tracer uptake. A simple intercurrent upper respiratory tract infection may make interpretation of enlarged, tracer avid, head and neck lymph nodes very difficult to differentiate from involvement from lymphoma. Furthermore, there may be a "flare response" following radiotherapy and chemotherapy, and postoperatively increased uptake may be evident particularly in the first 2-3 weeks. Thymic rebound characteristically appears in the 6-12 months after stopping myelosuppressive therapy, and is ${ }^{18}$ F-FDG avid. Granulocyte colony stimulating factor (G-CSF) therapy, which may be used when chemotherapy has resulted in severe myeloablation, may also give spurious results with increased uptake in much of the marrow. Accumulation of ${ }^{18}$ F-FDG in the kidneys and bladder may mask nearby pelvic and renal lesions, and its uptake by normal bone marrow makes differentiation from marrow diffusely infiltrated by, for example, neuroblastoma difficult. 
Furthermore, some tumours do not appear to accumulate ${ }^{18}$ F-FDG. ${ }^{17}$

The development of PET-CT scanners has enabled the radiologist to visualise both functional and anatomical data simultaneously, superimposed on a single screen. While CT scanning remains the most commonly used modality in clinical staging of lymphoma, nodal involvement is defined by an arbitrary increase in size $(1-2 \mathrm{~cm})$, which may fail to account for lymphomatous involvement of small nodes and may raise concerns about reactive enlarged nodes. Integration of functional and anatomic tumour characteristics provided by PET-CT imaging may be the most accurate non-invasive way of staging and monitoring paediatric lymphoma, and possibly other tumours.

While the potential for functional imaging and PET is clear, well designed prospective studies will be necessary as a matter of urgency; for example, for the paediatric lymphomas in particular, to define how best to utilise what is likely in the near future to remain a valuable but scarce resource, and to a lesser degree to understand better tracer avid non-malignant conditions which may confound interpretation of tumour activity. In the meantime, other forms of functional imaging such as MR spectroscopy are under development, with early studies showing that this may be of use in a number of paediatric tumours, although its use is only widespread in brain tumour imaging. ${ }^{18}$

\section{CONCLUSION}

In conclusion, it is clear that an understanding of the new imaging technologies, in addition to new ways of thinking about tumour staging and how tumours respond to therapy, will be essential for all clinicians involved in the management of paediatric tumours. Close collaboration with radiologists will be necessary, in order to make the best use of currently limited resources. Just as important, it must be remembered that PET and multislice CT in particular have the potential to dramatically increase the already large radiation burden in a population more likely to survive their primary cancer than ever before, and therefore they should be used sparingly and wisely.

\section{Authors' affiliations}

L Moon, Radiology Department, Barts and the London Hospitals NHS

Trust, London, UK

K McHugh, Radiology Department, Great Ormond Street Hospital for Children NHS Trust, London, UK

Competing interests: none declared

\section{REFERENCES}

1 Brenner D, Elliston C, Hall E, et al. Estimated risks of radiation induced fatal cancer from pediatric CT. AJR 2001;176:289-96.

2 WHO. Handbook for reporting results of cancer treatment. Geneva: World Health Organisation, 1979:48.

3 Therasse P, Arbuck S, Eisenhauer EA, et al. New guidelines to evaluate the response to treatment in solid tumours. J Natl Cancer Inst 2000:92:205-16.

4 McHugh K, Rao S. Response Evaluation Criteria In Solid Tumours (RECIST): problems and need for modifications in paediatric oncology. Br J Radiol 2003;76:433-6.

5 Husband JE, Schwartz LH, Spencer J, et al. Evaluation of the response to treatment of solid tumours - a consensus statement of the International Cancer Imaging Society. Br J Cancer 2004;90:2256-60.

6 Prokop M. Multislice CT: technical principles and future trends. Eur Radiol 2003; 13:M3-13.

7 Pappas J, Donnelly L, Frush D. Reduced frequency of sedation of young children with multisection helical CT. Radiology 2000;215:897-9.

8 Thomson G, Blair G. Multislice helical CT depiction of Wilms' tumor. J Pediatr Surg 2001;27:912-15.

9 Greess H, Lutze J, Nömayr A, et al. Dose reduction in subsecond multislice spiral CT examination of children by online tube current modulation. Eur Radiol 2004; 14:995-9.

10 Donnelly L, Emery K, Brody A, et al. Minimising radiation dose for pediatric body applications of single-detector helical CT. AJR 2001;176:303-6.

11 Warburg O. On the origin of cells. Science 1956;123:309-14.

12 Som $\mathrm{P}$, Atkins $\mathrm{HL}$, Bandopadhyay $\mathrm{D}$, et al. A fluorinated glucose analogue, 2-fluoro-2-D-glucose (F18):non-toxic tracer for rapid tumour detection. J Nud Med 1980;21:670-5.

13 Hudson M, Krasin M, Kaste S. PET Imaging in pediatric Hodgkin's lymphoma. Pediatr Radiol 2004;34:190-8.

14 Montravers F, Landman-Parker J, Grahek D, et al. Impact of FDG-PET on staging and management in childhood lymphoma: a three-year experience. Eur J Nucl Med Mol Imaging 2003;30(suppl 2):S194.

15 Shulkin B. PET imaging in pediatric oncology. Pediatr Radiol 2004; 34:199-204.

16 Bredella M, Caputo G, Steinbach L. Value of FDG positron emission tomography in conjunction with MR imaging for evaluating therapy response in patients with musculoskeletal sarcomas. AJR 2002;179:1145-50.

17 Shulkin B, Hutchinson R, Castle V, et al. Neuroblastoma: positron emission tomography with 2-[fluorine-18]-fluoro-2-deoxy-D-glucose compared with metaiodobenzylguanidine scintigraphy. Radiology 1996;199:743-50.

18 Vaidya S, Payne G, Leach M, et al. Potential role of MR spectroscopy in assessment of tumour response in childhood cancer. Eur $J$ Cancer 2003;39:728-35. 